\title{
EFFECT OF MAC DESIGN ON SOURCE ESTIMATION IN DENSE SENSOR NETWORKS
}

\author{
Min Dong and Lang Tong ${ }^{\dagger}$ \\ School of Electrical and Computer Engineering \\ Cornell University, Ithaca, NY 14853 \\ \{mdong,ltong\}@ece.cornell.edu
}

\author{
Brian M. Sadler \\ Army Research Laboratory \\ Adelphi, MD 20783 \\ bsadler@arl.army.mil
}

\begin{abstract}
We investigate the impact of medium access control (MAC) design on the reconstruction performance of a one-dimensional random signal field measured by a large scale sensor network. Assuming the sensor density goes to infinity, we show that MAC design affects the decay rate of reconstruction distortion, and thus the efficiency on reconstruction, as the number of received packets $M$ increases. Using a deterministic MAC with uniform spatial sampling, i.e., scheduling sensor transmissions from uniformly spaced locations, results in a faster decay rate of distortion than that using an ALOHA-like random access MAC. In particular, the ratio of the excess reconstruction distortion under random access MACs to that under the MAC with uniform sampling grows as $\log M+O(\log \log M)$. We further show that in the high measurement SNR regime, the benefit from carefully scheduling transmission instead of random access is substantial. In the low SNR regime, however, using random access MACs results in little reconstruction performance loss.
\end{abstract}

\section{INTRODUCTION}

Sensor network with mobile access points (SENMA) [1], shown in Fig. 1, is a network architecture for large scale low power sensor networks. In SENMA, sensors perform simple functions such as sensing / measurements. A small number of more powerful mobile nodes are responsible for collecting data from sensors and performing sophisticated signal processing. In some applications, sensors are densely deployed in the field to measure the signal of interest. At a prearranged time, sensors take local measurements forming a snapshot of the signal field. When the access point is ready to retrieve data, sensors transmit their packets back to the access point through a common channel according to a specified medium access control (MAC) protocol. Based on received data samples, the access point estimates the original signal field.

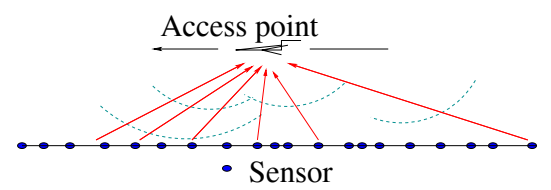

Fig. 1: a 1-D reachback sensor network with a mobile access point

The choice of MAC in SENMA can significantly affect the performance of signal reconstruction. If we ignore the quantization and measurement noise, receiving a packet from a sensor is equivalent to having the access point sample the signal field directly. For densely

${ }^{\dagger}$ This work was supported in part by the Multidisciplinary University Research Initiative (MURI) under the Office of Naval Research Contract N00014-00-1-0564, and Army Research Laboratory CTA on Communication and Networks under Grant DAAD19-01-2-0011. deployed sensors, data from neighboring sensors are highly correlated. Intuitively, collecting data from uniformly spaced sensors is better than collecting data from a concentrated sub-area because highly correlated measurements provide less information about the source. It is the MAC protocol that determines the positions of sensors from which data are successfully received.

If the mobile access point can schedule transmissions from sensors, it is natural to consider a deterministic MAC where uniformly spaced sensors are polled for their data. One such scheme is QUIRE [2]. Such a scheme ensures that data are collected from the best possible locations and no redundant transmissions are made. The deterministic MAC, however, comes with nontrivial complications of centralized scheduling. Furthermore, for finite sensor density, there may be regions void of sensors, which may result in substantial performance loss. (The problem of reconstruction with finite sensor density is considered in [3].) Decentralized random access such as ALOHA requires much less intervention from the mobile access point [4]. For such MACs, sensors transmit their packets probabilistically. The reception too is probabilistic, and packet collection can be modeled as random sampling of the signal field.

Implementation details aside, there is a fundamental question on how much gain (if any) the deterministic MAC has over random access. Should one carefully schedule sensor transmission in order to form the desired data sampling pattern, or simply allow random access? How does the performance gain/loss vary as more and more packets are collected?

In this paper, we consider signal field reconstruction using deterministic and random access MACs, with the maximum reconstruction distortion as the performance metric. To make the problem more tractable, we study the performance in a one-dimensional signal field, which provides insight into the two-dimensional problem. We use the optimal ${ }^{1}$ deterministic MAC that forms uniform sampling. We show that, as the number of received packets $M$ increases, this MAC scheme results in a faster decay rate of maximum reconstruction distortion than a random access MAC, so the deterministic MAC is more efficient on reconstruction. Specifically, the ratio of excess maximum distortion under random access to that under the MAC with uniform sampling grows as $O(\log M)$. We further show that in the high measurement SNR regime, the benefit from carefully scheduling transmission instead of random access is substantial. At low SNR, however, using random access MACs results in little reconstruction performance loss.

The MAC design problem for sensor networks has attracted a growing interest. Many MAC protocols have been proposed aiming to the special needs for both ad hoc sensor networks [5] and reachback sensor networks with access points [2,4]. Among these protocols, throughput and energy efficiency are the major considerations. How these MAC schemes actually affect the reconstruction performance has not been addressed. Perhaps the most relevant

\footnotetext{
${ }^{1}$ The optimality of uniform sampling is established in [3].
} 
work is [6], where the problem of sequential estimation of a dynamic source using random sampling in time is considered.

\section{PROBLEM STATEMENT}

\subsection{Source Model}

Consider a one-dimensional field of length $D$ denoted by $\mathcal{A}=$ $[0, D]$. Let $S_{t}(x)(x \in \mathcal{A})$ be the source of interest in $\mathcal{A}$ at time $t$. We assume that the spatial dynamic of $S_{t}(x)$ is a one-dimensional homogeneous Gaussian random field governed by the following linear stochastic differential equation:

$$
d S_{t}(x)=-f S_{t}(x) d x+\sigma d W_{t}(x)
$$

where $f$ and $\sigma \in \mathbf{R}$ are known, and $f>0^{2} . W_{t}(x)$ is a standard Brownian motion, and the source signal $S_{t}(x)$ is the stationary solution of (1) with $S_{t}(x) \sim \mathcal{N}\left(0, \frac{\sigma^{2}}{2 f}\right)$, for $x \in \mathcal{A}$. Furthermore, it can be shown that $S_{t}(x)$ is both Gaussian and Markovian. For $S_{t}(x)$ being homogeneous in $\mathcal{A}$, it has correlation: $E\left\{S_{t}\left(x_{0}\right) S_{t}\left(x_{1}\right)\right\}=$ $e^{-f\left(x_{1}-x_{0}\right)} \sigma^{2} / 2 f$, for $x_{0}<x_{1}$, which is only a function of distance between two points $x_{1}$ and $x_{0}$.

Suppose that a very large number of sensors are densely deployed in $\mathcal{A}$ for measurements. We denote the sensor density in $\mathcal{A}$ by $\rho$. We assume that the location of all sensors are known. During operation, all sensors take local measurements at a preprogrammed time $t$, and form a snapshot of the signal field. The measurement of a sensor at location $x$ and time $t$ is given by

$$
Y_{t}(x)=S_{t}(x)+N_{t}(x)
$$

where $N_{t}(x)$ is spatially independent and identically distributed (iid) zero mean white Gaussian measurement noise with variance $\sigma_{N}^{2}$, independent of $S_{t}(x)$. Note that we will reconstruct the source signal $\left\{S_{t}(x): x \in \mathcal{A}\right\}$ at time $t$ based only on the sensor measurements at time $t$. Therefore, we drop the time index for brevity in the following presentation.

\subsection{Two Types of MAC Schemes}

When the mobile access point is ready for data collection, sensors transmit packets, consisting of the measurement data and location information, to the access point through a common wireless channel. Sensors may contend for the channel or obey scheduling. Hence, we consider two types of MAC schemes: random access and deterministic.

For random access (such as ALOHA), sensors contend to access the channel with equal priority, and their packets have equal chance to get through. Under this type of MAC, the pattern of received $M$ packets origins, denoted as $\mathbf{P}_{M}=\left\{P_{1}, \cdots, P_{M}\right\}$, is random. This appears as if the access point randomly samples the sensor measurement data in $\mathcal{A}$. We denote this type of MAC scheme as $\pi_{r}$.

In contrast, a deterministic MAC scheme schedules transmissions according to a fixed pattern. In other word, the access point draws $M$ packets (samples) from predetermined locations. A special case is obtaining packets from uniformly spaced locations in $\mathcal{A}$. We refer to this as a MAC with uniform sampling, and denote it as $\pi_{u}$.

\footnotetext{
${ }^{2} f>0$, thus, (1) admits a stationary solution.
}

\subsection{Packet Retrieval}

Under the specified MAC scheme, after some collection time, the access point receives a total of $M$ packets originating from some $M$ points in $\mathcal{A}$. In other words, the access point obtains $M$ (noisy) samples of $S(x)$ in $\mathcal{A}$. To avoid the boundary effect for signal reconstruction, we assume that, during the data collection, the access point always obtains the packets from the two sensors closest to the two boundaries of $\mathcal{A}$. We denote the locations of these two "edge sensors" by $P_{0}$ and $P_{D}$. Fig. 2 shows an example of a resulting sampling of the signal field in $\mathcal{A}$. Based on these data samples, we then reconstruct the signal field.

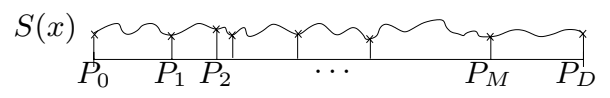

Fig. 2: a 1-D signal field sampled by a sensor network

\subsection{Source Estimation and Reconstruction Distortion}

Given the $M+2$ received packets (samples) from location $\mathbf{p}_{M}=$ $\left[P_{0}, P_{1}, \cdots, P_{M}, P_{D}\right]$, we denote the corresponding order statistics of the packet generation locations $\left\{P_{1}, \cdots, P_{M}\right\}$ by $P_{(1)}<\cdots<$ $P_{(M)}$. We estimate $S(x)$ at $x$ by MMSE smoothing using its two immediate neighbor samples: for $P_{(i)}<x<P_{(i+1)}$,

$$
\hat{S}(x)=E\left[S(x) \mid\left\{Y\left(P_{(i)}\right), Y\left(P_{(i+1)}\right)\right\}\right] .
$$

Notice that due to the Markov property of $S(x)$, in the noiseless measurement case, the above estimator is optimal in the sense that it results in the minimum MSE. In other words, the optimal MMSE estimator based on all received data samples reduces to (3). When measurement noise is present, the above estimator becomes suboptimal. Nonetheless, its simple structure and easy computation lends itself as an attractive practical estimator.

Let the measurement SNR be SNR $=\sigma^{2} / 2 f \sigma_{N}^{2}$. We define the maximum field reconstruction distortion given $\mathbf{p}_{M}$ by the maximum mean square estimation error in $\mathcal{A}$

$$
\mathcal{E}\left(\mathbf{p}_{M}, \mathrm{SNR}\right) \triangleq \max _{x \in \mathcal{A}} E\left\{|\hat{S}(x)-S(x)|^{2} \mid \mathbf{p}_{M}\right\} .
$$

A MAC scheme $\pi$ specifies how packets should be transmitted. It, therefore, determines how the signal field $\{S(x): x \in \mathcal{A}\}$ is sampled. Consequently, $\pi$ specifies the distribution of sample points $\mathbf{p}_{M}$. Under a given MAC scheme $\pi$, the average maximum distortion of the signal field is then given by

$$
\overline{\mathcal{E}}(M, \mathrm{SNR} ; \pi) \triangleq E\left\{\mathcal{E}\left(\mathbf{p}_{M}, \mathrm{SNR}\right) ; \pi\right\}
$$

where the expectation is taken over $\mathbf{p}_{M}$ for a given $M$.

Our goal is to analyze the signal field reconstruction performance under the two types of MACs. Specifically, we analyze how $\overline{\mathcal{E}}(M ; \pi)$ varies with $M$ and $\pi$.

\section{CALCULATING RECONSTRUCTION DISTORTION}

In this paper, we consider the case when sensor density goes to infinity, i.e., $\rho=\infty$. In this case, there exists a sensor at any point in $\mathcal{A}$. The two "edge" sensors are then at the two boundaries of $\mathcal{A}$, i.e., $P_{0}=0$ and $P_{D}=D$. As we have mentioned earlier, under random access MACs, the packet from each sensor has equal probability to get through. Therefore, given $M$ received 
packets $^{3}$ at the access point, their original locations $\left\{P_{1}, \cdots, P_{M}\right\}$ can be modeled as iid random variables with uniform distribution $U(0, D)$. In contrast, for deterministic MAC schemes, location points $\left\{P_{1}, \cdots, P_{M}\right\}$ are fixed. For the MAC with uniform sampling $\pi_{u}$, we have $\mathbf{P}_{M}=\left\{0, \frac{D}{M+1}, \frac{2 D}{M+1}, \cdots, D\right\}$.

\subsection{The Average Maximum Distortion}

By the Gaussian property of the process $\{S(x)\}$, the estimator in (3) is then given by

$$
\hat{S}(x)=E\left\{S(x) \mathbf{S}_{p}^{(i)^{H}}\right\}\left(E\left\{\mathbf{S}_{p}^{(i)} \mathbf{S}_{p}^{(i)}{ }^{H}\right\}+\sigma_{n}^{2} \mathbf{I}\right)^{-1} \mathbf{S}_{p}^{(i)}
$$

where $\mathbf{Y}_{p}^{(i)}=\left[Y\left(P_{(i)}, Y\left(P_{(i+1)}\right]^{T}, \mathbf{S}_{p}^{(i)}=\left[S\left(P_{(i)}\right), S\left(P_{(i+1)}\right)\right]^{T}\right.\right.$, and $P_{(i)}<x<P_{(i+1)}$. Finding $\mathcal{E}\left(\mathbf{p}_{M}, \mathrm{SNR}\right)$ in (4) can then be broken down to finding the maximum distortion of $S(x)$ in each interval $P_{(i)}<x<P_{(i+1)}$, for $i=0, \cdots, M$.

For $P_{(i)}<x<P_{(i+1)}$, we can show that the maximum MSE of $\hat{S}(x)$ is obtained at the middle point of $P_{(i)}$ and $P_{(i+1)}$, and it is only a function of the distance between $P_{(i)}$ and $P_{(i+1)}$. Moreover, it can be further shown that the maximum distortion $\mathcal{E}\left(\mathbf{p}_{M}, \mathrm{SNR}\right)$ in (4) is determined by the maximum of distances between any two adjacent data samples

$$
\mathcal{E}\left(\mathbf{p}_{M}, \mathrm{SNR}\right)=\frac{\frac{1}{\mathrm{SNR}}+1-e^{-f d_{\max }^{(M)}}}{\frac{1}{\mathrm{SNR}}+1+e^{-f d_{\max }^{(M)}}} \frac{\sigma^{2}}{2 f} \triangleq \mathcal{E}\left(d_{\max }^{(M)}, \mathrm{SNR}\right)
$$

where $d_{\max }^{(M)}=\max _{0 \leq i \leq M} d_{i}^{(M)}$. Then, the average maximum distortion in (5) is given by

$$
\overline{\mathcal{E}}(M, \mathrm{SNR} ; \pi)=E\left\{\mathcal{E}\left(d_{\text {max }}^{(M)}, \mathrm{SNR}\right) ; \pi\right\}
$$

where the expectation is now taken over $d_{\text {max }}^{(M)}$.

For deterministic MAC schemes, the reception pattern is fixed, and therefore $d_{\max }$ is fixed. We have

$\overline{\mathcal{E}}\left(M, \mathrm{SNR} ; \pi_{u}\right)=\mathcal{E}\left(\frac{D}{M+1}, \mathrm{SNR}\right)=\frac{\frac{1}{\mathrm{SNR}}+1-e^{-f \frac{D}{M+1}}}{\frac{1}{\mathrm{SNR}}+1+e^{-f \frac{D}{M+1}}} \frac{\sigma^{2}}{2 f}$.

In contrast, under random access MAC schemes, $d_{\text {max }}^{(M)}$ is random. To calculate the average distortion $\overline{\mathcal{E}}\left(M, \mathrm{SNR} ; \pi_{r}\right)$, we need to find the probability distribution of the maximum sample distance $d_{\max }^{(M)}$, denoted by $F_{d_{\max }}(x \mid M)$. It is given by the following [7]

$$
\begin{aligned}
& F_{d_{\max }}(x \mid M) \\
& =\left\{\begin{array}{lr}
0 & \text { if } 0 \leq x<\frac{D}{M+1} \\
g(M, x) & \text { if } \frac{D}{M-k+1} \leq x<\frac{D}{M-k}, k=0, \cdots, M-2 \\
1-(M+1)\left(1-\frac{x}{D}\right)^{M} & \text { if } \frac{D}{2} \leq x \leq D .
\end{array}\right.
\end{aligned}
$$

where

$$
g(M, x)=\sum_{i=0}^{k}(-1)^{i}\left(\begin{array}{c}
M+1 \\
i
\end{array}\right)\left[(M-i+1) \frac{x}{D}-1\right]^{M} .
$$

Using above and (8), $\overline{\mathcal{E}}\left(M, \mathrm{SNR} ; \pi_{r}\right)$ can be calculated by

$$
\overline{\mathcal{E}}\left(M, \mathrm{SNR} ; \pi_{r}\right)=\int \mathcal{E}(x, \mathrm{SNR}) d F_{d_{\max }}(x \mid M) .
$$

\footnotetext{
${ }^{3}$ The actual number of received packets is $M+2$. For convenience, we only count those packets not from the two boundaries of $\mathcal{A}$.
}

\subsection{The Estimation Distortion Ratio}

Notice that $d_{\max }^{(M)} \geq D /(M+1)$. It is clear that $\pi_{u}$ results in the minimum $\overline{\mathcal{E}}\left(M, \mathrm{SNR} ; \pi_{u}\right)$ among deterministic MAC schemes. We now compare the reconstruction performance under $\pi_{u}$ with that under $\pi_{r}$. Let $\overline{\mathcal{E}}(\infty, \mathrm{SNR} ; \pi)$ be the asymptotic distortion under a specific SNR and $\pi$ as the number of samples $M \rightarrow \infty$. It can be shown that $\overline{\mathcal{E}}\left(\infty, \mathrm{SNR} ; \pi_{r}\right)$ and $\overline{\mathcal{E}}\left(\infty, \mathrm{SNR} ; \pi_{u}\right)$ have the same expression given by

$$
\overline{\mathcal{E}}\left(\infty, \mathrm{SNR} ; \pi_{r}\right)=\overline{\mathcal{E}}\left(\infty, \mathrm{SNR} ; \pi_{u}\right)=\frac{\sigma^{2}}{2 f} \frac{1}{1+2 \mathrm{SNR}} .
$$

Having the same expression for asymptotic distortion under $\pi_{u}$ and $\pi_{r}$ is expected. As the number of samples goes to infinity, the asymptotic distortion is determined only by the measurement accuracy, i.e., the measurement SNR, not the specific MAC used.

Notice that as $M$ increases, the number of samples increases, and the maximum distortion under both types of MAC schemes decreases, but at different rates. Define the ratio of excessive maximum distortion under $\pi_{r}$ to that under $\pi_{u}$ as

$$
r(M, \mathrm{SNR}) \triangleq \frac{\overline{\mathcal{E}}\left(M, \mathrm{SNR} ; \pi_{r}\right)-\overline{\mathcal{E}}\left(\infty, \mathrm{SNR} ; \pi_{r}\right)}{\overline{\mathcal{E}}\left(M, \mathrm{SNR} ; \pi_{u}\right)-\overline{\mathcal{E}}\left(\infty, \mathrm{SNR} ; \pi_{u}\right)} .
$$

Because $d_{\max }^{(M)}$ is the smallest under $\pi_{u}$, we have $r(M$, SNR $) \geq 1$. From (9) and (10), $r(M, \mathrm{SNR})$ can be calculated for any given $M$. To ease the computation, we can also use bounds on $r(M, \mathrm{SNR})$, based on the following inequality.

$$
\begin{aligned}
& (\mathcal{E}(D, \mathrm{SNR})-\mathcal{E}(0, \mathrm{SNR})) x / D \leq \mathcal{E}(x, \mathrm{SNR})-\overline{\mathcal{E}}\left(\infty, \mathrm{SNR}, \pi_{r}\right) \\
& \leq x \sigma^{2}(1 / \mathrm{SNR}+1) /(1 / \mathrm{SNR}+2)^{2}
\end{aligned}
$$

The bounds on $r(M, \mathrm{SNR})$ can then be calculated using (10), (12), and (13). As a function of $M, r(M, \mathrm{SNR})$ shows the difference of the decreasing rate under $\pi_{r}$ and $\pi_{u}$.

\section{ASYMPTOTIC BEHAVIOR OF DISTORTION RATIO}

We now analyze the asymptotic behavior of distortion ratio $r(M)$ as $M$ increases. We have the following.

Theorem 1 For reconstructing the one-dimensional Gaussian random signal field described in (1) in a sensor network, as $M \rightarrow \infty$, the ratio of the excessive maximum estimation distortion $r(M, S N R)$ defined in (12), is given by

$$
r(M, S N R)=\log M+O(\log \log M) .
$$

Theorem 1 shows that the ratio of excessive maximum distortion under $\pi_{r}$ to that under $\pi_{u}$ has a logarithmic growth rate. This in turn tells us that the MAC design affects the decreasing rate of reconstruction distortion as the number of received packets increases, and therefore the efficiency of the reconstruction. Using the MAC with uniform sampling $\pi_{u}$ results in the fastest decreasing rate of the distortion over $M$. This MAC efficiency can also be interpreted in another way. The following corollary tells us how many packets the access point needs under each type of MAC to reach the same reconstruction distortion level.

Corollary 1 Let $M_{\pi_{u}}$ and $M_{\pi_{r}}$ be the number of packets needed under $\pi_{u}$ and $\pi_{r}$ respectively, at the access point, to reconstruct the signal field with the same level of distortion. Then

$$
M_{\pi_{u}}=O\left(\frac{M_{\pi_{r}}}{\log M_{\pi_{r}}}\right) .
$$




\subsection{Reconstruction Performance in Different SNR Regimes}

As we have mentioned, the MAC with uniform sampling $\pi_{u}$ is optimal in the sense that it results in the minimum signal reconstruction distortion. We now compare the actual distortion performance $\overline{\mathcal{E}}(\infty, \mathrm{SNR} ; \pi)$ in different SNR regimes. Define

$$
v(M, \mathrm{SNR})=\frac{\overline{\mathcal{E}}\left(M, \mathrm{SNR} ; \pi_{r}\right)}{\overline{\mathcal{E}}\left(M, \mathrm{SNR} ; \pi_{u}\right)} .
$$

$v(M, \mathrm{SNR})$ (in $\mathrm{dB})$ indicates the performance gap between $\pi_{r}$ and $\pi_{u}$.

As SNR $\rightarrow \infty$, i.e., the noiseless measurement case, we have

$$
\overline{\mathcal{E}}\left(\infty, \mathrm{SNR} ; \pi_{r}\right)=\overline{\mathcal{E}}\left(\infty, \mathrm{SNR} ; \pi_{u}\right)=0 .
$$

In this case, from Theorem 1 , as $M \rightarrow \infty$, we have

$$
v(M, \infty)=r(M, \infty)=\log M .
$$

This indicates that the reconstruction performance gap between $\pi_{r}$ and $\pi_{u}$ increases. Therefore, in the high SNR regime, as the number of received packets becomes large, the MAC with uniform sampling $\pi_{u}$ provides a large performance gain over the random access MAC. The benefit from carefully scheduling transmission instead of random access is substantial.

In the low SNR regime, the value of $\overline{\mathcal{E}}(\infty, \mathrm{SNR} ; \pi)$ is large. As $M$ increases, $\overline{\mathcal{E}}(M, \mathrm{SNR} ; \pi)$ decreases and is soon saturated to $\overline{\mathcal{E}}(\infty, \mathrm{SNR} ; \pi)$, and therefore $v(M, \mathrm{SNR}) \rightarrow 1$. This demonstrates that, when the measurement SNR is low, despite the lower efficiency of the random access MAC, it results in very little reconstruction performance loss, compared with $\pi_{u}$. In this regime, the reconstruction performance is dominated by the measurement noise, and is less affected by the choice of different MAC schemes.

\subsection{Numerical Results}

Fig. 3 plots the average maximum distortion and the distortion ratio vs. $M$, respectively. We set $f=0.2, \sigma^{2}=1$, and $D=5$. Fig. 3 (top) shows the upper and lower bounds of distortion ratio $r(M, \mathrm{SNR})$ vs. $M$, under $\pi_{r}$ and $\pi_{u}$ respectively, in the noiseless case. The bounds are calculated using (13). Besides the bounds, the dotted line plots the scaled $\log M$ using the lower bound of (14). We observe that, with $M$ greater than 200, the growth rate of $r(M, \infty)$ is already approximately $\log M$, matching the asymptotic behavior of $r(M, \infty)$ in Theorem 1 .

Fig. 3 (bottom) shows the distortion curves $\overline{\mathcal{E}}(M, \mathrm{SNR} ; \pi)$ vs. $M$ at $\mathrm{SNR}=10 \mathrm{~dB}$ and $20 \mathrm{~dB}$, respectively. We see that the distortion performance under $\pi_{u}$ is sensitive to the noise level. The performance gap under the two different MAC schemes becomes smaller as the measurement noise become higher.

\section{CONCLUSIONS}

In sensor networks, the choice of MAC can significantly affect the performance of signal reconstruction. For reconstructing a onedimensional signal field, assuming the sensor density goes to infinity, we have shown that the MAC with uniform sampling $\pi_{u}$ provides higher efficiency on reconstruction, i.e., it results in faster decay rate of distortion as the number of received packets $M$ increases. In particular, the ratio of excessive distortion under random access MAC $\pi_{r}$ to that under $\pi_{u}$ grows as $O(\log M)$. For high measurement SNR, this translates to a substantial performance gain of $\pi_{u}$ over $\pi_{r}$. However, we have shown that, when the measurement noise is high, the difference of the actual reconstruction distortion between $\pi_{u}$ and $\pi_{r}$ becomes small. At low SNR, random access results in little reconstruction performance loss. Notice that for finite sensor density, there may not exist sensors at desired locations, therefore performance under $\pi_{u}$ may suffer from missing samples. Using random access in this case may result in better performance. How the reconstruction is affected in this case is discussed in [3].
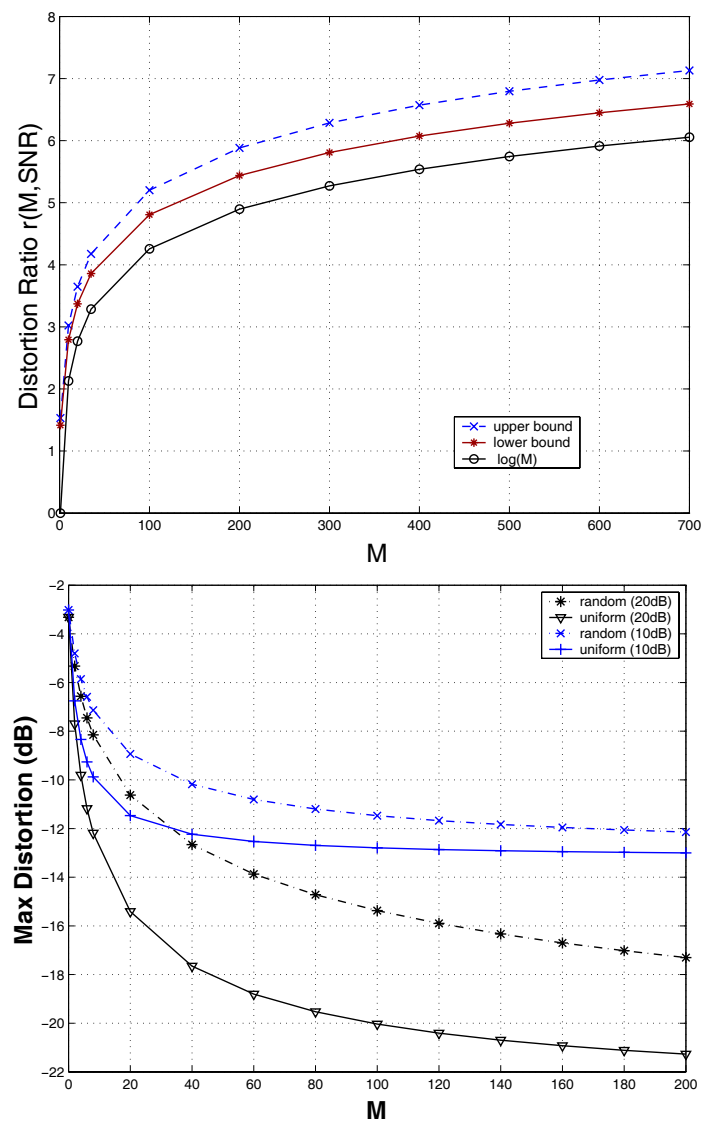

Fig. 3: $f=0.2, \sigma^{2}=1$, and $D=5$. Top: Distortion ratio vs. $M$ (noiseless). Bottom: Distortion vs. $M$.

\section{REFERENCES}

[1] L. Tong, Q. Zhao, and S. Adireddy, "Sensor Networks with Mobile Agents," in Proc. 2003 MILCOM, (Boston, MA), Oct. 2003.

[2] Q. Zhao and L. Tong, "QoS Specific Information Retrieval for Densely Deployed Sensor Network," in Proc. IEEE 2003 MILCOM, Oct. 2003.

[3] M. Dong, L. Tong, and B. Sadler, "Impact of MAC design on estimation of spatial Markov process in sensor networks." to be submitted to IEEE Trans. on Signal Processing, 2003.

[4] P. Venkitasubramaniam, S. Adireddy, and L.Tong, "Sensor Networks with Mobile Agents: Optimal Random Access and Coding," submitted to IEEE Journal on Sel. Areas in Comm., July 2003.

[5] R. Iyer and L. Kleinrock, "QoS Control for Sensor Networks," in Proc. of 2003 ICC, May 2003.

[6] M. Micheli and M. I. Jordan, "Random Sampling of a Continuous-time Stochastic Dynamical System," in Proc. of the 15th Int. Sym. on MTNS 2002, Aug. 2002.

[7] M. Dong, L. Tong, and B. M. Sadler, "Source reconstruction via mobile agents in sensor networks: throughput-distortion characteristics," in Proc. IEEE MILCOM, Oct. 2003. 